\title{
Manifestations of Metacognitive Activity during the Collaborative Planning of Chemistry Practical Investigations
}

\author{
Kgadi Mathabathe $^{\mathrm{a} 1}$ and Marietjie Potgieter ${ }^{\mathrm{a}}$ \\ ${ }^{a}$ University of Pretoria, South Africa
}

This paper elaborates a process followed to characterise manifestations of cognitive regulation during the collaborative planning of chemistry practical investigations. Metacognitive activity was defined as the demonstration of planning, monitoring, control and evaluation of cognitive activities by students while carrying out the chemistry task. Inherent in collaborative learning is the social aspect of metacognition, which in this study was evidenced in social cognitive regulation (notably of intra-and inter-personal metacognitive regulations) as groups of students went about planning their practical investigations. Discussions of two of the learning groups $(n=$ 4; $n=3$ ) as they planned the extended practical investigation were recorded, transcribed and analysed for indicators of any inherent metacognitive activity. The process of characterising the manifestations of metacognition resulted in the development of a coding system which specifies not only the regulatory strategies at play but the type of regulation (self or other), the area of regulation (cognition, task performance or behavior) as well as the depth of regulatory contributions (high or low). The fine-grained coding system allowed for a finer theoretical elucidation of the social nature of metacognition. The implications of this study for metacognition and chemistry education research are highlighted.

Keywords: Social regulation, Collaborative learning, Metacognitive regulation, Chemistry laboratory instruction

\footnotetext{
${ }^{1}$ Corresponding author. University of Pretoria. Department of Science, Mathematics and Technology Education, Corner Leyds and George Storrar Street, Groenkloof, 0181, South Africa. E mail: kgadi.mathabathe@up.ac.za
} 


\section{Introduction}

Metacognition can simply be defined as thinking about thinking. Educational psychologists have consistently emphasised the importance of metacognition for supporting student learning (Brown, 1987; Flavell, 1979). Much research has resulted in rich descriptions of metacognitive activity as it pertains to solving mathematics problems (Iiskala, Vauras, \& Lehtinen, 2004) and studying for reading comprehension (Koch, 2001), but little is known about its role and how it applies in the chemistry laboratory when students try to plan for an investigation, master practical manipulations and regulate experimental conditions. Some attempts have been made to study metacognition in chemistry laboratory contexts e.g. Davidowitz and Rollnick (2003) and Sandi-Urena, Cooper and Stevens (2012).

Davidowitz and Rollnick (2003) carried out a case study to investigate the role of a model called the Competency Tripod model and flow diagrams in enhancing metacognition with regard to laboratory work. Flow diagrams proved to be highly successful as metacognitive tools. SandiUrena et al. (2012) conducted a mixed methods study to investigate the effect of cooperative problem-based laboratory instruction on metacognition and problem solving. Students were found to display improved problem solving skills as well as increased cognitive regulation skills. Both studies were conducted with the aim of investigating the role the interventions played in enhancing students' metacognition. As in the case of these two studies the present study was carried out during an intervention in a laboratory context designed to engage students in active and deep learning. However, the focus of the present study was not to investigate the impact of the intervention but to identify and characterise manifestations of metacognitive activity inherent in the collaborative planning of practical investigations. 
Metacognition is embedded in scientific inquiry because successful inquiry requires scientists to constantly reflect on and direct their thinking towards the desired outcome. The development of metacognitive competencies is a desired outcome in science education because it promotes meaningful learning, autonomy and self-regulation (Kipnis \& Hofstein, 2008). This study formed part of a bigger project for revamping third year organic chemistry laboratory instruction by the replacement of recipe based laboratory work with a simulated industrial project that required collaborative inquiry. This study was conducted to answer the research question: How does metacognitive activity manifest during the collaborative planning of a chemistry practical investigation?

This paper provides a detailed description of the process that was followed to analyse students' group discussions for manifestations of metacognitive activity. Researchers have looked at the role that the introduction of various teaching and learning interventions play in enhancing metacognitive skills. However, to my knowledge very few science education researchers have looked into the metacognitive activity inherent in the verbal communication indicative of these regulatory processes in natural settings. In future, the characterisation of metacognitive activity developed in this study may serve as a useful tool for assessing the extent to which the desirable skills of metacognitive regulation are elicited by the interventions put in place.

\section{Metacognition}

The commonly used definition of metacognition states that it is an individual's knowledge and regulation of thinking (Schraw, Crippen, \& Hartley, 2006). Knowledge of cognition is about being aware of one's thinking, the kind of learner one is and about the nature of the task. Regulation of cognition on the other hand has to do with how individuals monitor and regulate their thinking while performing a task. Regulation of cognition which is the primary focus of this 
study is characterised by a set of activities that help students monitor and control their thinking while completing a task. Although a number of regulatory activities have been identified in the literature, four activities or skills are widely recognised: planning, monitoring, control and evaluation (Lai, 2011).

Planning, also described as forethought by Pintrich (2000), involves projecting forward regulating thinking regarding the strategies for optimal task performance, allocation of resources, setting of goals, roles and responsibilities, and clarifying task demands and expectations ahead of task execution (Khosa \& Volet, 2014; Schraw et al., 2006; Whitebread et al., 2009). Monitoring is characterised by checking and assessing thinking or understanding in connection with content, resources, procedures and strategies that are necessary for task performance (Schraw et al., 2006). The behaviours associated with monitoring serve as a means for comparing understanding and performance against standards or learning goals (DiDonato, 2013).

Control is defined as a regulatory process that shifts the cognitive flow toward optimal task performance or conceptual understanding (Khosa \& Volet, 2014). It is further characterised by behaviour that is expressed with the intention of influencing the way an individual has been thinking to enhance task performance. Evaluation includes actions taken to appraise learning processes, as well as the products of task performance. It is usually characterised by evaluative statements or judgements that we make about our thinking, understanding and task performance (Pintrich, 2000). In this study we were interested in how these regulatory activities identified in the context of self-regulated problem solving manifested as students were planning chemistry practical investigations collaboratively. 


\section{The Social Aspect of Metacognition}

Previously studies of metacognition in learning contexts have dealt with metacognitive regulation in purely individualistic terms (Iiskala et al., 2004). However, recent studies have shown convincingly that cognitive regulation observed at the individual level can be further enhanced through collaborative problem solving activities. This notion draws from Vygotsky (1978)'s theory of proximal development that an individual's ability to complete a task optimally can be enhanced with peer collaboration. We share King's (1998) understanding of true collaboration as entailing more than the effective division of labour consistent with cooperative learning, but rather meeting the criteria of comparable expertise, interactivity, interdependence and reciprocity in interaction and activity amongst team members (Vauras, Iiskala, Kajamies, \& Lehtinen, 2003).

Social interaction supports the development of metacognitive skills in multiple ways. Peers can play the role of communicative partners making us aware of our thoughts and requiring us to articulate our thoughts more clearly (Fox \& Riconscente, 2008). Whitebread et al. (2009) argue that instances of social regulation are supported by interactions during collaborative learning because working in a group results in a reduction in cognitive load, allowing participants to monitor and regulate the reciprocal use of the joint understanding of the task. Social interaction inherent in collaborative tasks obliges participants to make explicit and articulate their thoughts and conceptions to others (Iiskala et al., 2004). Metacognitive activity can be inferred from these verbalised thoughts and conceptions (Whitebread et al., 2009). To this end many studies have shown that peer interaction inherent in collaborative learning activities can have positive effects on the development of metacognitive skills of individuals in a group (Chan, 2012; Grau \& Whitebread, 2012). 
A review of the literature reveals that Socially Shared Metacognitive Regulation (SSMR), a term coined by Iiskala et al. (2004) often manifests as self, other and shared regulation. Selfregulation is observed when an individual monitors and controls his/her own thinking (Whitebread et al., 2009). Other-regulation is observed in a situation in which one partner masters a key element of the task but the other(s) do(es) not, so that one partner instructs the other(s)" (Whitebread et al., 2009, p. 67). Finally, Iiskala et al. (2004) describe shared regulation as joint monitoring and regulation of the task by members of a team. Next, we discuss the context of the study, the sample selected for participation as well as the process followed for data analysis.

\section{Methodology}

Collaborative problem solving is characterised by discussions and negotiations among team members while individual problem solving consists of the thoughts and utterances by one individual. Therefore, the social dynamics inherent in collaborative problem solving distinguish indicators of social regulation as requiring a coding system that captures instances of intra- and interpersonal cognitive regulation. Several coding systems have been designed to capture instances of social regulation at the intra- and inter-individual levels (e.g. DiDonato, 2013; Khosa \& Volet, 2014). However, metacognitive activities often remain covert mechanisms taking place inside the head of the students (Veenmann, van Hout-Wolters, \& Afflerbach, 2006) which complicates their direct observation. Systematic observation and think aloud protocols are some of the on-line methods that have been successfully used by researchers to observe individual and social metacognitive regulation in action (Azevedo, 2009). Systematic observation was deemed a suitable method for the purposes of investigating manifestations of metacognitive activity in the current study. 


\section{Context}

Tasks that combine elements of collaboration and ill-structured inquiry based learning have proved to be effective activities that not only improve science learning but develop the metacognitive lifelong learning skills needed at higher levels of science (Sandi-Urena et al., 2012). These instructional strategies stimulate cognitive conflict and require deep thinking as opposed to the simple recall of straightforward answers. Students have to enter into negotiations, monitoring and steering of each other's thinking towards the correct answer in the context of such collaborative tasks. Negotiations anticipated during collaborative problem solving means that the researchers interested in studying socially mediated regulation have to identify and distinguish between instances of intra- and inter-individual regulation.

The context of this study is a simulated industrial project with an intricate design which formed part of senior undergraduate laboratory training. The project was designed to model what new graduates are likely to experience when moving into an industrial environment. Students were required to take on the role of professional chemists working for a hypothetical company. The company had identified an opportunity to produce and market an organic chemical compound. However, there were three possible synthetic routes that could be used to produce the compound. Each route consisted of three synthetic steps using different starting materials and chemical reactions, but leading to the same final product of which a fixed quantity $(2 \mathrm{~g})$ had to be produced. The chemists were tasked with evaluating the three routes experimentally and advising management on which route was the most cost effective, environmentally friendly and least technically challenging (Pilcher, Riley, Mathabathe, \& Potgieter, 2015). The next paragraphs focus on aspects of the industrial project that pertain to this paper. 
The simulated industrial project combined elements of guided inquiry, collaborative learning, contextualisation and metacognitive prompts in the form of reflective learning strategy questionnaires (RLSQs). In keeping with the jigsaw group work approach (Aronson, 2000), students were placed in home and specialist groups. In the home groups, each of three students were given the responsibility of evaluating one of the synthetic routes experimentally and giving feedback to his/her home group, assisting the group to make an informed decision and formulate a final recommendation. First, students had to extrapolate from the given condensed experimental procedures and generate their own detailed procedures in preparation for the laboratory trials. To do this, a structured planning session was scheduled where members of home groups dispersed into specialist groups of 4 or 5 made up of students from other teams allocated the same synthetic route.

Specialist groups made use of the resources at their disposal such as a condensed experimental procedure, materials safety data sheets (MSDS), suppliers catalogues etc. and extrapolated the detailed procedures, relevant safety data, glassware, equipment as well as amounts required to carry out the synthesis in the laboratory. Specialist group discussions took up about three hours of the four-hour planning session. The instructor and teaching assistant took on the role of facilitators, clarifying task requirements, answering any questions students had and probing them to think harder by asking leading questions. As the specialist groups completed their tasks, the instructor and teaching assistant would check the work to make sure that each group could safely proceed to the laboratory. This form of facilitation ensured that the all the students designed a safe, working procedure and calculated appropriate quantities.

Conceptual understanding in Chemistry and more so in the planning of chemistry practical investigations requires students to operate simultaneously at the macroscopic, submicroscopic 
and symbolic levels of thinking (Johnstone, 1991). Requiring students to calculate quantities of reagents and to generate their own experiment procedures made the task even more challenging. Successful completion of authentic inquiry based tasks (Chinn \& Malhotra, 2002) requires students to engage in deep thought and metacognitive activity. Adding an element of collaboration reduced cognitive load allowing participants to engage in the monitoring and regulation of the joint understanding of the task.

Most studies have looked at academic achievement scores or performance as a measure of the success of metacognition training (Aghaie \& Zhang, 2012). In our study, determining the quality of feedback and regulatory contributions that the students made towards predicting the best route after working in their specialist groups served as an indication of whether or not the combined implicit (collaborative inquiry-based laboratory design) and explicit (metacognitive prompts) eliciting of cognitive and metacognitive activity resulted in learning gains and understanding of the task.

\section{Participants}

The study was conducted with a consenting group of third year organic chemistry students $(n=$ 39). Both the Monday $(n=20)$ and Thursday $(n=19)$ planning sessions consisted of seven home groups and six specialist groups. The allocation of students into home and specialist groups was based on the assessment of the students' abilities in previous laboratory activities as well as on peer-friend relationships within the student body. The first author was present in both sessions as a participant observer.

Because the analysis of the data relied heavily on making inferences from the specialist group discussions, it was necessary to interview the participants afterwards to provide confirming or disproving evidence (Miles, Huberman, \& Saldana, 2014). A selection of cases for in-depth 
analysis was therefore made on the basis of all of the team members being willing to participate in the follow-up focus group interviews. Recorded discussions of two specialist groups while planning investigations were purposively chosen for in-depth analysis. Although discussions by both specialist groups were analysed in-depth, only excerpts from Team Kagiso, named after its team leader will be used to illustrate how the verbal discussions in this context were analysed for manifestations of metacognitive activity.

\section{Team Kagiso}

This study was conducted in South Africa, a multicultural and multiracial nation with 11 official languages. Thus, participants in this study constituted a multiracial class of students from different cultural backgrounds and home languages, receiving instruction in English. Team Kagiso consisted of four members - three Black males, Kagiso, Amos and Leonard and one Black female, Reneilwe. During the specialist group discussions, Kagiso emerged as vocal and confident and he spontaneously assumed a leadership position. This was welcomed by all group members as it seemed that they all thought highly of him and trusted his chemistry content knowledge.

While Kagiso and Leonard were observed to be the academically strong members of the team the two members were different in how they regulated cognitive activities. Kagiso was assertive while Leonard was tentative in his interaction and regulatory efforts. Reneilwe and Amos relied heavily on Kagiso and Leonard with their engagement characterised by a lack of confidence and a constant need for validation. These dynamics are brought to the fore because we believe that they shaped the metacognitive engagement in this group. In deciding how to tackle the task, the group decided to split duties. Kagiso and Amos were responsible for deciding on the types and sizes of glassware to be used, as well as the safety data. Reneilwe and Leonard worked on the 
calculations of reagents and had to supply Kagiso and Amos with the mass and volume information necessary for decisions regarding suitable glassware.

\section{Data Analysis}

Although task instructions were communicated in English the students were free to carry out specialist group discussions in a language of their choice. Students in team Kagiso carried out their group discussions primarily in Setswana, one of the 11 official languages in South Africa and the primary language of the group members. Although this meant that language would not be a barrier in allowing students to make explicit their thoughts and thought processes, it resulted in a lot of code switching between English and Setswana. Being well conversant in Setswana the first author could translate all conversations into English after transcription. However, to validate the translations the accuracy of translated conversations was assessed by forward and back translation of the turns of talk by a fellow researcher who is also first language Setswana speaker. In the event of discrepancies we engaged in discussion and adjusted the translations accordingly.

Team Kagiso's specialist group discussion was transcribed and translated word for word by the first author. The sequence of discussions were numbered as turns of talk. A turn began when an individual took the stage in a conversation, and ended when another person took over (Hogan, 1999). Team Kagiso's specialist group discussion resulted in 2920 turns of talk including verbal contributions by the lecturer, neighbouring students, researchers and teaching assistants. Students' verbal contributions alone constituted 2618 turns of talk. To simplify the process of coding, each turn of talk was assigned a single code. All turns of talk were coded, none was skipped. With the complexity of naturally occurring social talk, we observed some turns of talk 
which could not be classified as clear-cut metacognitive or non-metacognitive. Statements requiring dual coding as metacognitive and non-metacognitive were coded twice, however, in deciding on the encompassing code for the purposes of identifying metacognitive turns, priority was given to the regulatory contributions that the statements were making. A good example is the following utterance made by Leonard in the initial stages of the specialist group discussions:

8. LEONARD: Do we have to speak loud or not really? No, we need a specialist group thing! (referring to the RLSQ).

The first part of the turn in bold was interpreted as a simple non-regulatory question with Leonard establishing the acceptable rules of engagement and the second part as a regulatory statement with Leonard alerting his team mates to missing information. While going through the task instructions Leonard realized that they needed to simultaneously complete the reflective learning strategy questionnaire (RLSQ) and alerted the team that they needed to obtain one from the instructor before proceeding with the task. In coding the turn was classified as regulatory.

From the self-regulation literature we anticipated that cognitive regulation manifests in activities of planning, monitoring, control and evaluation as students solve a problem (Brown, 1987; Flavell, 1979; Pintrich, 2000). From the social regulation literature we understood that in a social context cognitive regulation manifests as self, other and shared regulation (Iiskala et al., 2004). However, in the current study we focused only on manifestations of self- and other-regulation which enabled a cleaner characterisation of the data. The process of analysing verbal discussions for manifestations of metacognitive activity started off with a partial theory of what constitutes cognitive regulation in collaborative group discussions as planning, monitoring, control, and evaluation, with these activities occurring at the intra- (self) and inter-individual levels (other). 
The turns of talk in the form of either questions, assertions, instructions, judgements or suggestions from which planning, monitoring, control or evaluation could be inferred were interpreted as regulatory. The inclusion or exclusion of statements in these categories was dependent on whether the statements aimed at influencing and steering individuals' thinking. The following decision tree gives an overview of the decisions that guided the process of classifying students' utterances into cognitive regulatory activities of planning, monitoring, control and evaluation.

Being an internal mechanism, metacognition is generally inferred from students' verbal and nonverbal indicators. Inferences from non-verbal behaviour have been particularly useful in studying cognitive regulation by young children with limited verbal proficiency (Whitebread et al., 2009). With our participants constituting senior undergraduate students we focused only on the manifestations of metacognitive activity emerging from the students' verbal expressions. For this purpose, we had to reconceptualise the definitions of the different components of cognitive regulation to be consistent with the focus of this study. Planning was thus inferred from any verbalisation demonstrating forethought by interrogating the thinking prior to task execution. Monitoring was inferred from any verbalisation characterised by the checking of thinking about the task. Control was inferred from any verbalisation that seemed to have been expressed with an intention to influence thinking, usually to improve or enhance task execution. Lastly, evaluation was inferred from any verbalisation that was characterised by evaluative statements or judgements made about thoughts related to the task. We opted to use the term 'manifestations' of regulation to refer to the way in which the cognitive regulation activities of planning, monitoring, control, and evaluation were revealed during group discussions. 
A further distinction in terms of the types of regulation (self- and other-regulation) was made. Intrapersonal regulation (self) was inferred from statements that were made to influence the student's own thinking during task execution. Interpersonal regulation (other) was inferred from statements directed at influencing the thinking of a fellow team member or the group as a whole. Appendix I supplements Figure 1 to further illustrate the inclusion and exclusion criteria used to characterise verbal expressions into the different categories of metacognitive activity.

Excerpts from team Kagiso are presented next to demonstrate how social regulation was inferred from the team's verbalisations. Words in the primary language of the students are presented in italics with English translations in parenthesis. The manifestations of regulation were indicated by the following acronyms: planning (PLAN), monitoring (MON), control (CTRL), and evaluation (EVAL). Other-regulation and self-regulation were indicated by OR and SR respectively.

\section{Planning/Forethought}

Evidence of forward thinking was observed in excerpts of planning shown below.

Example PLAN1: A few minutes into the specialist group discussion Leonard posed this question trying to put forth a strategy to optimise task performance: 
183. LEONARD: how would you like to split it? (the task) Someone does MSDS (Materials Safety Data Sheets), someone does the calculation, someone proposes the apparatus and someone proposes how the actual experiment can be done what do you think?

This turn was coded as: [PLAN_OR] - this statement is a demonstration of forward thinking because through this question Leonard was clarifying the logistics of task performance before commencing with task execution. By asking "how would you like to split it?" and "what do you think?" Leonard was establishing what his peers thought about the best way to go about performing the task which made this a metacognitive question stimulating his peers' thinking, thus other-regulatory.

Example PLAN2: Reneilwe and Leonard had been given the task of performing calculations and determining amounts of reagents required. Leonard would perform the calculations and Reneilwe would supply him with the chemical data like the molar masses, densities, etc. of substances for use in the calculations. In this example Reneilwe verbalised how she planned to optimise her own task performance, i.e. by writing the data on the side, while she waited for Leonard to get to a step where he needed the data to finalise the calculation.

841. RENEILWE: Ke ireng Leonard? ke go biletse ntho e la (?) le moo ke kereile (?) ke go botse density ya teng? (what should I do Leonard? Should I call out that thing for you (?) I have also found that one (?) should I tell you its density?) [...]

847. RENEILWE: (?) ko ngwala mo thoko (I will write it on the side)

Turn 847 was coded as: [PLAN_SR] -Anticipating that she would be ahead of Leonard, she thought ahead and determined that she would find the chemical data and write it down while she waited for him to finish what he was doing. Hence, this statement demonstrated planning on her part. This was interpreted as self-regulation as her comment seemed to have been expressed to regulate her own task performance strategy. 


\section{Monitoring}

Monitoring manifested mostly as expressions in the form of questions asked with the intention to check own or peers' comprehension of task instructions, or to seek validation of own thinking by peers or sometimes by the instructor.

Example MON1: Frustrated Leonard checked if his fellow team members read up on what the desired product was used for. He asked this question to try and put his point across that once the team knew what the product was used for they could extrapolate the experimental procedure from that information.

116. LEONARD: okay what is this product used for? What is that for? Did you read it? You didn't read it?

117. KAGISO: it's not on the (?) [...]

120. LEONARD: $J a$ (Yes) but then the first thing we research is what is that thing used for. Google it quickly in your phone. So if it is used for, for consumption by people or whatever, do you get that? 'cause that's very helpful. It means that you are thinking about it along the right line. I think I am saying too much and I am gonna give you guys a headache.

Turn 116 was coded as: [MON_OR] - This turn was interpreted as other-monitoring as Leonard checked if his team mates had done research on the desired product and further on in turn 120, tried to convince them that reading up on what the desired product was used for is also crucial information for optimal task performance.

Example MON2: In response to Kagiso's suggestion in turn 210, Leonard checks whether Kagiso understands and realises what the prompt (What will you do in order to compile the detailed experimental procedure for the synthetic route, i.e. distribution of tasks - who will do what?) in the RLSQ asks them to do: 
210. KAGISO: we just know the product that we need to form. So then $n n a$ (myself) I am suggesting that each, we all work on the mechanism and once that we had, we compare it and we know gore (that) this is the route that we are taking. Then work out wena (you) you can find the MSDS, wena (you) you can work out the experimental procedure...

211. LEONARD: but then that's what they are asking us now, do you realise that? Please would you read it for us? If you don't mind please.

Turn 211 was coded as: [MON_OR] - This question was interpreted as other-monitoring as Leonard was checking whether Kagiso realised that what he had suggested as a way forward was actually what the prompt had asked them to do.

\section{Control}

Control of individual and peer cognition was observed in instances when individuals attempted to enhance task performance by seeking clarification from peers or lecturer, to explain to their peers in an attempt to change their minds or to correct their peers' thinking.

Example CTRL1: Kagiso and Amos realised that they did not understand something about the solution they needed to use and they asked the instructor for clarification:

743. AMOS: (reads from the summarised experimental procedure) and the solution was washed with water?

744. KAGISO: mm (agrees) (?) this doesn't makes sense. Dr P! Dr P! [...] (calls the lecturer)

748. AMOS: eh madam! (Kagiso laughs) [...]

750. AMOS: um we don't understand here, (reads) the residue was dissolved in dichloromethane and the solution was washed with water. Which solution? The one we (?) stirred overnight or the residue solution?

Turn 750 was coded as: [CTRL_SR] - this statement was interpreted as self-control because it was clear from the context that Amos consulted with the instructor to make sure he understood instructions correctly before conveying the instructor's explanation to the team.

Example CTRL2: While reading through the condensed experimental procedure Amos came across an instruction to dissolve the residue, but he was confused because based on his 
understanding residue is a substance that should be discarded. Kagiso addressed this misconception.

735. AMOS: ke nako, twenty four hours byanong re etsa eng? (it's time, twenty four hours, what do we do now?) (reads) The residue was dissolved in...residue byang? Residue ke matlakala moes? (what do they mean residue? Isn't residue rubbish?)

736. KAGISO: no the remainder. When you transfer it.

Turn 736 was coded as: [CTRL_OR] - this statement was interpreted as other-regulation as Kagiso corrects Amos' understanding and clarifies the concept of residue in chemical terms.

\section{Evaluation}

Evaluation was inferred from any verbalisation that was characterised by evaluative statements or judgements made about own or peers' thoughts related to the task. Evaluative statements that exemplified this category are provided next.

Example EVAL1: Halfway through the specialist group discussions, after Kagiso explained to her, Reneilwe made an evaluative statement about her understanding of the logic behind the calculations they have done so far.

1524. KAGISO: akere ke (isn't it) fifty percent so you need twice that what you need, you need twice of it and for this one $k e$ (it's) fifty percent you need twice of that so it's forty eight point four millimoles (?) and for that it's twice the yield so you need eighty (?) do you follow my logic?

1525. RENEILWE: now I get it (the calculation) ne kentse ke re why e ya twice why e seng half we're working back (?) (now I get it I was thinking to myself why it goes twice why not half we're working back)

Turn 1525 was coded as: [EVAL_SR] - This statement was interpreted as self-evaluation because only later on Reneilwe realised why they had performed calculations the way they did and she expressed this as an evaluative statement of her own understanding. 
Example EVAL2: In the excerpt below Leonard was responding to the instructor's questions about how he thought the group could proceed in their attempt to generate detailed experimental procedures from the given information.

319. LECTURER: Okay so then I, apart from the main hazards, how? Where do you think you should start? Now. Can you de... (determine) eh how do you know what glassware to use? How big, how small? What glassware to use? [...]

321. LECTURER: what chemicals so you've got the chemicals there.

322. LEONARD: and mass what volume

323. LECTURER: what mass and what volume, how are you gonna get what mass and what volume?

324. LEONARD: that's the question we had 'cause we don't know the efficiency of every step and...

Turn 324 was coded as: [EVAL_OR] - This statement was interpreted as other-evaluation because Leonard was not only making a judgement about his own knowledge of the task but the team as a whole.

The process of classification and coding of manifestations was cyclical and evolutionary as the limitations of existing theoretical understanding became evident. The initial codes adapted from the literature ( (Khosa \& Volet, 2014; Whitebread, et al., 2009) did not make a distinction between instances when students regulated thinking about the underlying chemistry concepts, correct understanding of the language of the task, i.e. task instructions and requirements. Classifying verbalisations as either planning, monitoring, control or evaluation and as either intra- or interpersonal was not sensitive enough to pick up nuances in these manifestations. The development of an all-encompassing coding system suitable for the unique context of a chemistry laboratory as the outcome and contribution of the study will be described next. 
A review of the literature on self-regulated learning led to the work of Pintrich (2000) which validated our observations that classifying cognitive regulation required a system that also stipulates the areas where the individuals apply their regulatory efforts. Pintrich (2000) speaks about areas of regulation which constitute cognition about the content, task features, behaviour and task performance. The area of regulation was an additional dimension that was added to the classification system to make the coding more comprehensive. Using codes that could capture the manifestation, type and area of regulation allowed for a coding system that would be sensitive to the subtle differences that existed in the verbalisations exemplary of metacognitive regulation in this context.

Areas of regulation emerged inductively from the data and were coded as follows: cognition (COGN), behaviour (BEHAV) and task performance (TASK). Two additions, COGN(C) and $\operatorname{COGN}(\mathrm{T})$ were used to distinguish between the regulation of chemistry(C) related cognition(COGN) and the regulation of task(T) related cognition (COGN). Thus the codes assigned to statements indicated the manifestation, type, and area of regulation, as well as the sub-codes in parentheses serving as brief descriptions of the verbalisations unique to the context of chemistry laboratory instruction, e.g. MON_OR_COGN(C) (checks peer's understanding about the chemistry content). Examples demonstrating how the excerpts provided above were further classified are presented next. 


\section{Planning/Forethought}

Leonard's regulatory efforts in turn 183 were interpreted as directed towards influencing his team mates' thinking about task performance while turn 847 was interpreted as Reneilwe regulating her own task performance.

\section{Example PLAN1}

183. LEONARD: how would you like to split it? Someone does MSDS, someone does the calculation, someone proposes the apparatus and someone proposes how the actual experiment can be done what do you think?

[PLAN_OR_TASK] (proposes strategy to optimise task performance)

\section{Example PLAN 2}

847. RENEILWE: (?) ko ngwala mo thoko (I will write it on the side)

[PLAN_SR_TASK] (proposes strategy to optimise own task performance)

\section{Monitoring}

In example MON1 Leonard's verbalisations were interpreted as monitoring as he checked whether his peers had researched the uses of the desired product. In example MON2 Leonard's question was interpreted as him checking his peer's understanding of the task requirements.

\section{Example MON1}

116. LEONARD: okay what is this product used for? What is that for? Did you read it? You didn't read it?

[MON_OR_TASK] (checks peer's task performance)

\section{Example MON2}

211. LEONARD: but then that's what they are asking us now, do you realise that? Please would you read it for us? If you don't mind please.

[MON_OR_COGN(T)] (checks peer's understanding of task instructions) 


\section{Control}

All the excerpts shown below were interpreted as regulatory efforts applied to influence thinking about the underlying chemistry concepts. The difference is that example 1 was interpreted as a regulatory effort directed at the self while that of example 2 was directed at the peer.

\section{Example CTRL1}

750. AMOS: um we don't understand here, (reads) the residue was dissolved in dichloromethane and the solution was washed with water. Which solution? The one we (?) stirred overnight or the residue solution?

[CTRL_SR_COGN(C)] (seeks clarification from the lecturer)

\section{Example CTRL2}

736. KAGISO: no the remainder. When you transfer it.

[CTRL_OR_COGN(C)] (clarifies peer's thinking about the chemistry)

\section{Evaluation}

In example EVAL1, Reneilwe's judgement was based on her understanding of the task requirements. In example EVAL2, Leonard was interpreted as making an evaluative statement about the knowledge of the team as a whole.

\section{Example EVAL1}

1525. RENEILWE: now I get it ne kentse ke re why e ya twice why e seng half we're working back (?) (I was thinking to myself why it goes twice why not half we're working back)

[EVAL_SR_COGN(T)] (makes judgement about own understanding of the task)

\section{Example EVAL2}

324. LEONARD: that's the question we had 'cause we don't know the efficiency of every step and...

[EVAL_OR_COGN(C)] (makes judgement about group knowledge) 


\section{Depth of regulation}

The final step of refinement of the coding scheme was prompted by the realisation that the scheme did not make explicit the fact that some of the regulatory contributions were more successful than others in fostering the carrying out of the task with understanding. Each manifestation was then evaluated for depth of regulation as determined by whether the regulatory contributions fostered critical thinking and conceptual understanding of the individual or the group. The framework of socially regulated learning developed by Volet, Summers and Thurman (2009) and the work of Khosa and Volet (2014) were helpful in the process of delineating verbal expressions as indicative of high- or low-level regulation.

High-level regulation was inferred from instances when the students established conceptual understanding by seeking explanation (Seek meaning: SM), volunteering an explanation (Volunteer meaning: VM), providing conceptual justification (CJ), and stimulating thinking (ST). Low-level regulation was inferred from instances when the students sought to acquire information (Seek Information: SI), give information (GI), and instigate regulation without offering conceptual justification (noCJ).

The excerpt from team Kagiso below is one example where students demonstrated high-level regulation. The discussion started with Reneilwe reading from the RLSQ and indicating to her team members the first metacognitive prompt that they needed to tackle having read the brief. In turns 81,83 and 85 below, Leonard tried to sway peers from opting for a simplistic way of thinking about the task by urging them to think deeper before writing down the answer and this behaviour was interpreted as high-level interpersonal regulation. 
68. RENEILWE: [reads from RLSQ] upon completion of this task return to the home groups and present the information. So this is what we answer before. [reads from $R L S Q]$ What information, oh! after reading this what information is missing? how will we obtain it? What will you do in order to compile the synthetic route? [...]

75. KAGISO: It says "what information is missing? How will you obtain this information?"

76. RENEILWE \& LEONARD: Okay.

77. KAGISO: Google it [instructs peer].

78. AMOS: Sure Kagiso (?) reference resources.

79. RENEILWE: Obviously the quantities, everything the quantities, apparatus is missing.

80. AMOS: But akere (isn't it) we know we have to get at least a product of 2 grams so we just work from there, backwards.

81. LEONARD: No no no that's, if you want to work backwards it means that you have to have the efficiency of every single step [emphasising by hitting the table with pen].

82. AMOS: No they say at least 2 grams.

83. LEONARD: $J a$ (Yes) but then...

84. RENEILWE: Okay guys what information is missing?

85. LEONARD: If you need at least 2 grams then you need the efficiency of every single step [emphasising again]. [...]

Low-level regulation was observed in instances when students simply provided their peers with information without offering an explanation to improve their understanding or when individuals simply accepted whatever answer was provided by their peers without requiring conceptual justification. This form of engagement was observed in the verbal interaction between Kagiso and Amos below.

808. AMOS: separating funnel, the whole solution after the chloro methane e sala mo separationeng ne (remains in the separating funnel right)?

809. KAGISO: mm [agrees] this is your separating funnel.

In the next paragraphs we discuss the process followed to establish trustworthiness for the coding system. 


\section{Establishing Trustworthiness for the Coding System}

Two rounds of peer debriefing (Lincoln \& Guba, 1985) were used to establish the consistency, dependability and credibility of the system of coding. Two colleagues, experienced in qualitative research but with no direct experience with our research, served as analytic audience, one during the development of the coding system and the other upon completion of the first cycle of coding. Discussions held daily over a period of one week led to consensus about the definitions and the verbalisations indicative of the different aspects of social regulation.

During the second round of peer debriefing another colleague was given the specialist group discussion transcript, coding scheme as well as directions for coding and asked to act as independent coder. The colleague coded a portion of student talk consisting of two pages of transcript. The two pages of transcript consisted of 26 turns of talk, 14 of which were interpreted as non-metacognitive and 12 of which were regarded as metacognitive statements. The level of inter-coder agreement was determined by calculating Cohen's Kappa, a statistic used in similar research to determine inter-rater reliability (Lippmann Kung \& Linder, 2007). Six out of 12 metacognitive turns were coded the same which resulted in a Cohen's kappa value of 0.35, indicating a poor level of agreement (Landis \& Koch, 1977).

Setswana was not the primary language of the independent coder which made it difficult for her to relate to the language dynamics in the turns of talk. Thus, most of the disagreements arose because of a particular type of statement that was perceived to have dual meaning. Statements such as 'it is this one, right?' could be interpreted as either a clarification seeking statement (control) or a validation seeking statement (monitoring). Having a good understanding of the cultural undertones and the language dynamics the first author was able to explain that the statements in a form of a question ending with the word 'right' were representative of validation 
seeking behaviour and therefore a monitoring strategy used by the students used to get their peers or instructors to confirm their thinking. The verbalisation indicated a tendency of seeking validation from the leader which is common cultural practice. Disagreements encountered for metacognitive statements were resolved through discussion to achieve better agreement. Two conflicting turns remained unchanged, which resulted in a Cohen's kappa of 0.75 , indicating good agreement (Fleiss, 1981).

\section{Discussion}

This study was conducted to answer the research question: How does metacognitive activity manifest during the collaborative planning of a chemistry practical investigation? Our findings concurred with literature reports of studies in other contexts by providing evidence for social regulation as manifesting in the form of planning, monitoring, control and evaluation, both at the intra- and inter-individual levels (Iiskala et al., 2004; Khosa \& Volet, 2014; Whitebread et al., 2009). However, it became clear that reporting our observations only in terms of these four components of regulation and their social dimension would not portray the richness of metacognitive activity in an undergraduate chemical laboratory. The context-specific nature of the data required further classification in terms of areas of regulation, an aspect of social regulation that no other literature accounts prepared us for. Allowing this aspect of social regulation to inductively emerge from the data generated themes that specified the regulation of thinking about different facets of the activity, namely task performance (TASK), behaviour (BEHAV), task features [COGN(T)] as well as the underlying chemistry concepts [COGN(C)]. We believe that researchers wishing to duplicate our study in the same domain will find these insights particularly useful. To those wishing to carry out a similar study in a different domain we advise to allow such insights to emerge from their data. 
The final iteration of our description of the manifestations of metacognitive activity in the collaborative planning of practical investigations was guided by the work of Volet and coworkers (Khosa and Volet, 2014; Volet et al., 2009) and consisted of a value judgement on the quality or depth of regulation. We found this additional layer of analysis to be particularly useful in distinguishing between students in terms of their unique styles of regulation in a social context. Using a data analysis system that could capture the manifestation, type, area and depth of regulation allowed for characterisation that was comprehensive enough to identify and describe the subtle differences that existed in the manifestations of metacognitive activity in a discipline-specific social context. Such a comprehensive description of the manifestations of metacognitive activity in a particular context should enable instructional designers to monitor whether learning environments specifically designed to develop metacognitive skills are indeed achieving their goals.

Carrying out this type of research had its fair share of challenges. Naturally occurring talk does not fit neatly into turns and categories. Transcribing group discussions was not easy, especially during episodes of turns of talk where members spoke over each other or when they were not sufficiently audible. The strategy to have each team member introduce themselves at the start of the recording helped us to recognise each student's voice while listening to the recording. The students' verbalisations were better understood in context as opposed to isolated turns of talk. Coding episodes of group engagement instead of individual turns of talk (Volet et al., 2009) could be used in future as an alternative approach to study the conversations of the groups in depth.

Our observation was that a fine line exists between monitoring and control behaviours. Clear distinctions between behaviours associated with each of the manifestations of metacognitive 
regulation remains a methodological challenge in this area of research. This observation may be explained by the fact that metacognitive activity is a covert mechanism and evidence of its existence is largely dependent on subjective inferences made by researchers. High level of inference however, poses a challenge to reliability (Whitebread et al., 2009) and difficulties in reaching consensus about the behaviours that exemplify the different manifestations between researchers and independent coders is a cause for concern (Whitebread et al., 2009).

Our findings served to illustrate the multifaceted nature of discipline-specific social regulation. Similar findings were reported by Grau and Whitebread (2012) in a study in which they sought to identify aspects of social regulation demonstrated by young children in collaborative science activities. In this study we have developed a decision tree for the classification of verbalisations (Figure 1) and a set of detailed criteria for including or excluding verbalisations in each of the categories of classification (Appendix I). We consider these tools as our contribution to researchers wishing to document or monitor the manifestation of metacognitive activity in similar or different collaborative learning contexts.

\section{Acknowledgements:}

For funding: National Research Foundation (South Africa), Canon Collins Trust and Legal administered Ros Moger Terry Furlong scholarship, Association for Commonwealth Universities, University of Pretoria postgraduate research support.

\section{References}

Aghaie, R., \& Zhang, L. (2012). Effects of explicit instruction in cognitive and metacognitive reading strategies on Iranian EFL students' reading performance and strategy transfer. Instructional Science, 40(6), 1063-1081. doi:10.1007/s11251-011-9202-5

Aronson, E. (2000). Jigsaw Classroom. Retrieved from http://www.jigsaw.org/index.html 
Azevedo, R. (2009). Theoretical, conceptual, methodological, and instructional issues in research on metacognition and self-regulated learning: A discussion. Metacognition and Learning, $4(1), 87-95$.

Brown, A. L. (1987). Metacognition, executive control, self-regulation, and other more mysterious mechanisms. In F. E. Weinert, R. H. Kluwe, F. E. Weinert, \& R. H. Kluwe (Eds.), Metacognition, motivation, and understanding (pp. 65-116). Hillsdale, NJ: Lawrence Erlbaum Associates.

Chan, C. K. (2012). Co-regulation of learning in computer-supported collaborative learning environments: a discussion. Metacognition and Learning, 7(1), 63-73.

Chinn, C. A., \& Malhotra, B. A. (2002). Epistomologically authentic inquiry in schools: a theoretical framework for evaluating inquiry tasks. Science Education, 86(2), 175-218.

Davidowitz, B., \& Rollnick, M. (2003). Enabling metacognition in the laboratory: a case study of four second year university chemistry students. Research in Science Education, 33, 4369.

DiDonato, N. C. (2013). Effective self- and co-regulation in collaborative learning groups: an analysis of how students regulate problem solving of authentic interdisciplinary tasks. Instructional Science, 41, 25-47.

Flavell, J. H. (1979). Metacognition and cognitive monitoring: a new area of cognitive developmental inquiry. American Psychologist, 34(10), 906-911.

Fleiss, J. (1981). Statistical methods for rates and proportions (2 ed.). New York: John Wiley. 
Fox, E., \& Riconscente, M. (2008). Metacognition and self-regulation in James, Piaget, and Vygotsky. Educational Psychology Review, 20(4), 373-389.

Grau, V., \& Whitebread, D. (2012). Self and social regulation of learning during collaborative activities in the classroom: the interplay of individual and group cognition. Learning and Instruction, 22(6), 401-412.

Hogan, K. (1999). Relating students' personal frameworks for science learning to thier cognition in collaborative contexts. Science Education, 83(1), 1-32.

Iiskala, T., Vauras, M., \& Lehtinen, E. (2004). Socially-shared metacognition in peer learning? Hellenic Journal of Psychology, 1, 147-178.

Johnstone, A. H. (1991). Why is science difficult to learn? things are seldom what they seem. Journal of Computer Assisted Learning, 7(2), 75-83.

Khosa, D. K., \& Volet, S. E. (2014). Productive group engagement in cognitive activity and metacognitive regulation during collaborative learning: can it explain differences in students' conceptual understanding? Metacognition and Learning, 9(3), 287-307.

King, A. (1998). Transactive peer tutoring: distributing cognition and metacognition. Educational Psychology Review, 10(1), 57-74.

Kipnis, M., \& Hofstein, A. (2008). The inquiry laboratory as a source for development of metacognitive skills. International Journal of Science and Mathematics Education, 6(3), 601-627.

Koch, A. (2001). Training in metacognition and comprehension of physics texts. Science Education, 85(6), 758-768. 
Lai, E. R. (2011). Metacognition: a literature review. Pearson.

Landis, J. R., \& Koch, G. G. (1977). The measurement of observer agreement for categorical data. Biometrics, 33(1), 159-174.

Lincoln, Y. S., \& Guba, E. G. (1985). Naturalistic Inquiry. Thousand Oaks: CA: Sage Publications.

Lippmann Kung, R., \& Linder, C. (2007). Metacognitive activity in the physics student laboratory: is increased metacognition necessarily better? Metacognition and Learning, 2(1), 41-56.

Miles, M. B., Huberman, A. M., \& Saldana, J. (2014). Qualitative data analysis: a methods sourcebook (3 ed.). Thousand Oaks, CA: Sage.

Pilcher, L. A., Riley, D. L., Mathabathe, K. C., \& Potgieter, M. (2015). An inquiry-based practical curriculum for organic chemistry as preparation for industry and postgraduate research. South African Journal of Chemistry, 68, 236-244.

Pintrich, P. R. (2000). The role of goal orientation in self-regulated learning. In M. Boekaerts, P. R. Pintrich, \& M. Zeidner (Eds.), Handbook of Self-regulation (pp. 451-502). San Diego: Elsevier.

Sandi-Urena, S., Cooper, M. M., \& Stevens, R. H. (2012). Effect of cooperative problem-based lab instruction on metacognition and problem-solving skills. Journal of Chemical Education, 89(6), 700-706. 
Schraw, G., Crippen, K. J., \& Hartley, K. (2006). Promoting self-regulation in science education: metacognition as part of a broader perspective on learning. Research in Science Education, 36(1), 111-139.

Vauras, M., Iiskala, T., Kajamies, A. K., \& Lehtinen, E. (2003). Shared-regulation and motivation of collaborating peers: a case analysis. Psychologia, 46, 19-37.

Veenmann, M. V., van Hout-Wolters, B. H., \& Afflerbach, P. (2006). Metacognition and learning: conceptual and methodological considerations. Metacognition and Learning, 1, $3-14$.

Volet, S., Summers, M., \& Thurman, J. (2009). High-level co-regulation in collaborative learniing: How does it emerge and how is it sustained? Learning and Instruction, 19, 128 - 143. doi:10.1016/j.learninstruc.2008.03.001

Vygotsky, L. S. (1978). Mind and society: the development of higher mental processes. Cambridge, MA: Harvard University Press.

Whitebread, D., Coltman, P., Pasternak, D. P., Sangster, C., Grau, V., Bingham, S., .. . Demetriou, D. (2009). The development of two observational tools for assessing metacognition and self-regulated learning in young children. Metacognition and Learning, 4(1), 63-85. 
Appendix I. Inclusion and exclusion criteria for coding statements as indicative of the different aspects of domain-specific social regulation

\begin{tabular}{|c|c|c|}
\hline Manifestations of regulation & Inclusion criteria & Exclusion criteria \\
\hline Planning (PLAN) & $\begin{array}{l}\text { This category includes verbalisations that demonstrate forethought by } \\
\text { interrogating thinking about planning e.g. clarifying own or peers' thinking } \\
\text { regarding task instructions, critiquing suggestions for optimal task execution } \\
\text { prior to task execution. }\end{array}$ & $\begin{array}{l}\text { This category excludes verbalisations made to acquire task-related or general } \\
\text { information from instructors or peers prior to task execution and not necessarily } \\
\text { made to regulate planning related thinking. }\end{array}$ \\
\hline Monitoring (MON) & $\begin{array}{l}\text { This category includes verbalisations characterised by checking of thinking } \\
\text { about underlying chemistry concepts, strategies for optimal task performance, } \\
\text { conducive behaviour etc. } \\
\text { The verbalisations associated with monitoring serve as a means for establishing } \\
\text { understanding and thinking against learning goals (DiDonato, 2013). }\end{array}$ & $\begin{array}{l}\text { This category excludes verbalisations made to check all other aspects of the task } \\
\text { except thinking about the underlying chemistry concepts, task features, task } \\
\text { performance or conducive behaviour. }\end{array}$ \\
\hline Control (CTRL) & $\begin{array}{l}\text { This category includes turns of talk characterised by verbalisations that shift the } \\
\text { cognitive flow by influencing thinking toward optimal task performance or joint } \\
\text { understanding of the task (Khosa \& Volet, 2014). }\end{array}$ & $\begin{array}{l}\text { This category excludes verbalisations made to influence all other aspects of the } \\
\text { task except thinking about the underlying chemistry concepts, task features, task } \\
\text { performance or conducive behaviour. }\end{array}$ \\
\hline Evaluation (EVAL) & $\begin{array}{l}\text { This category included verbalisations made to evaluate thinking about the task, } \\
\text { subject matter, task performance or conducive behaviour. }\end{array}$ & $\begin{array}{l}\text { This category excludes verbalisations made to evaluate all other aspects of the } \\
\text { task except thinking about the underlying chemistry concepts, task features, task } \\
\text { performance or conducive behaviour. }\end{array}$ \\
\hline \multicolumn{3}{|l|}{ Types of regulation } \\
\hline Self-regulation (SR) & $\begin{array}{l}\text { This category included statements that were made to influence student's own } \\
\text { thinking. }\end{array}$ & $\begin{array}{l}\text { This category excluded verbalisations that were made to influence the thinking } \\
\text { of a fellow team member or the team as a whole. } \\
\text { This category also excluded instances of shared regulation observed in instances } \\
\text { of joint regulation of the task by team members (Iiskala et al., 2004). }\end{array}$ \\
\hline Other-regulation (OR) & $\begin{array}{l}\text { This category included verbalisations that were made to influence the thinking } \\
\text { of a fellow team member or the team as a whole. }\end{array}$ & $\begin{array}{l}\text { This category excluded statements made to influence student's own thinking. } \\
\text { This category also excluded instances of shared regulation observed in instances } \\
\text { of joint regulation of the task by team members (Iiskala et al., 2004). }\end{array}$ \\
\hline \multicolumn{3}{|l|}{ Area of regulation } \\
\hline $\begin{array}{l}\text { Regulation of thinking about the } \\
\text { task }[\mathrm{COGN}(\mathrm{C})]\end{array}$ & $\begin{array}{l}\text { This category includes statements made to regulate thinking about the } \\
\text { underlying chemistry concepts. }\end{array}$ & $\begin{array}{l}\text { This category excludes statements made to regulate thinking about all other } \\
\text { aspects of the task not related to the chemistry content. }\end{array}$ \\
\hline $\begin{array}{l}\text { Regulation of thinking about the } \\
\text { underlying chemistry concepts } \\
{[\mathrm{COGN}(\mathrm{T})]}\end{array}$ & $\begin{array}{l}\text { This category includes statements made to regulate thinking related to the task } \\
\text { features, e.g. instructions }\end{array}$ & $\begin{array}{l}\text { This category excludes statements made to regulate thinking about all other } \\
\text { aspects of the task not related to task features. }\end{array}$ \\
\hline $\begin{array}{l}\text { Regulation of task performance } \\
\text { (TASK) }\end{array}$ & $\begin{array}{l}\text { This category includes statements made to regulate thinking about optimal task } \\
\text { performance. }\end{array}$ & $\begin{array}{l}\text { This category excludes statements made to regulate thinking about all other } \\
\text { aspects of the task not related to task performance. }\end{array}$ \\
\hline $\begin{array}{l}\text { Regulation of conducive } \\
\text { behaviour (BEHAV) }\end{array}$ & $\begin{array}{l}\text { This category includes statements made to regulate thinking about what } \\
\text { constitutes conducive behaviour. }\end{array}$ & $\begin{array}{l}\text { This category excludes statements made to regulate thinking about all other } \\
\text { aspects of the task not related to what constitutes conducive behaviour. }\end{array}$ \\
\hline \multicolumn{3}{|l|}{ Depth of regulation } \\
\hline Low-level regulation (LL) & $\begin{array}{l}\text { This category includes statements made to monitor or regulate thinking by } \\
\text { requiring information or giving information without justification to promote } \\
\text { conceptual understanding. }\end{array}$ & $\begin{array}{l}\text { This category excludes statements made to establish or foster critical thinking } \\
\text { and conceptual understanding by seeking explanation, volunteering an } \\
\text { explanation, providing conceptual }\end{array}$ \\
\hline High-level regulation (HL) & $\begin{array}{l}\text { This category includes statements made to establish or foster critical thinking } \\
\text { and conceptual understanding by seeking explanation, volunteering an } \\
\text { explanation, providing conceptual justification, and stimulating thinking. }\end{array}$ & $\begin{array}{l}\text { This category excludes statements made to monitor or regulate thinking by } \\
\text { requiring information or giving information without conceptual justification. }\end{array}$ \\
\hline
\end{tabular}


Figure 1. Decision tree showing the decisions that informed the classification of verbalisations into the different cognitive regulation activities

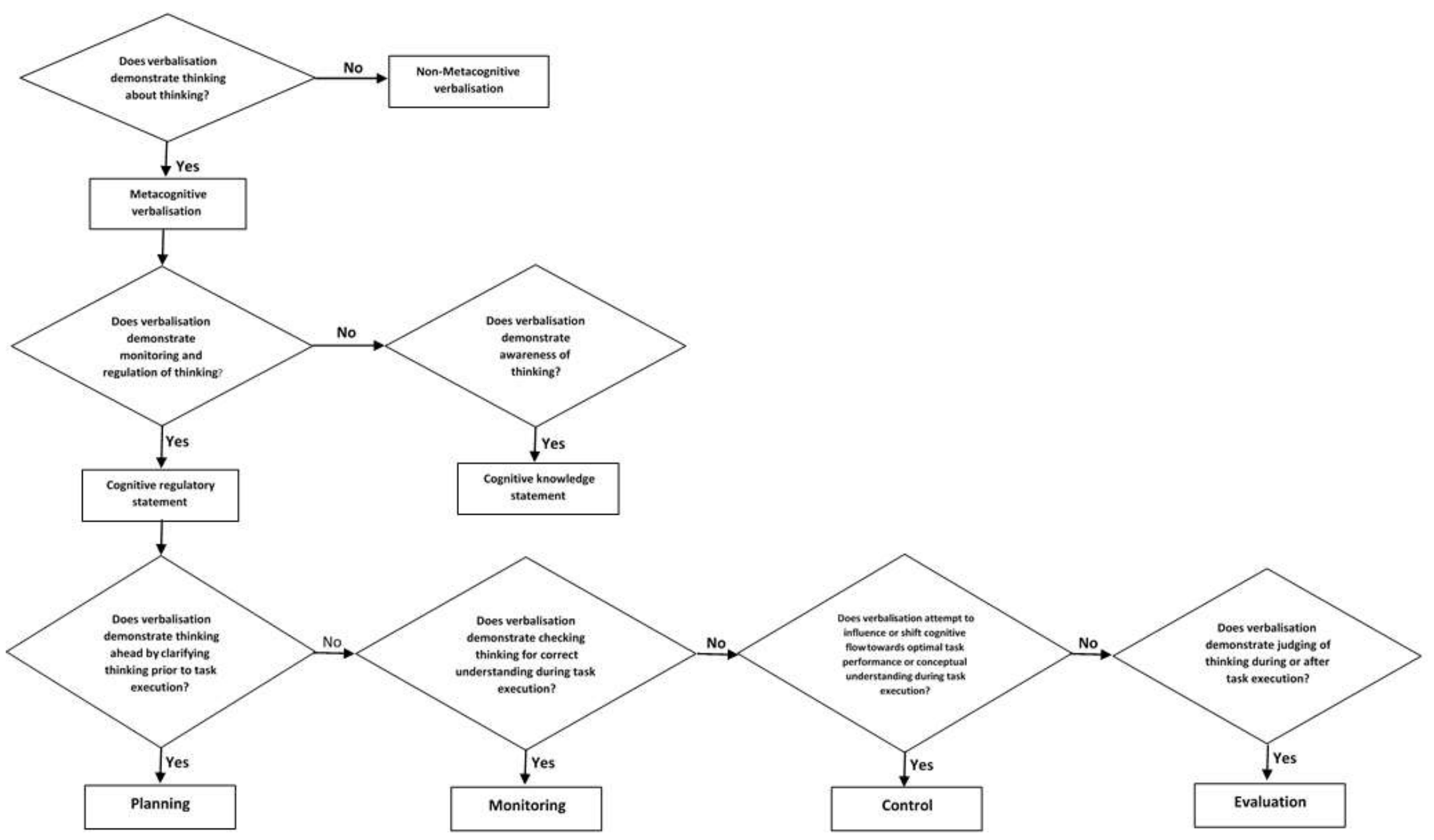

